\title{
Lattice constant in nonstoichiometric uranium dioxide from first principles
}

\author{
Fabien Bruneval \\ DEN, Service de Recherches de Métallurgie Physique, CEA, Université Paris-Saclay, F-91191 Gif-sur-Yvette, France \\ Michel Freyss \\ CEA, DEN, DEC, Centre de Cadarache, 13108 Saint-Paul-lez-Durance, France \\ Jean-Paul Crocombette \\ DEN, Service de Recherches de Métallurgie Physique, CEA, Université Paris-Saclay, F-91191 Gif-sur-Yvette, France
}

(Received 24 November 2017; published 2 February 2018)

\begin{abstract}
Nonstoichiometric uranium dioxide experiences a shrinkage of its lattice constant with increasing oxygen content, in both the hypostoichiometric and the hyperstoichiometric regimes. Based on first-principles calculations within the density functional theory (DFT) $+U$ approximation, we have developed a point defect model that accounts for the volume of relaxation of the most significant intrinsic defects of $\mathrm{UO}_{2}$. Our point defect model takes special care of the treatment of the charged defects in the equilibration of the model and in the determination of reliable defect volumes of formation. In the hypostoichiometric regime, the oxygen vacancies are dominant and explain the lattice constant variation with their surprisingly positive volume of relaxation. In the hyperstoichiometric regime, the uranium vacancies are predicted to be the dominating defect,in contradiction with experimental observations. However, disregarding uranium vacancies allows us to recover a good match for the lattice-constant variation as a function of stoichiometry. This can be considered a clue that the uranium vacancies are indeed absent in $\mathrm{UO}_{2+x}$, possibly due to the very slow diffusion of uranium.
\end{abstract}

DOI: 10.1103/PhysRevMaterials.2.023801

\section{INTRODUCTION}

Uranium dioxide, the most prevalent nuclear fuel over the globe, exists for a wide range of stoichiometries around the ideal $\mathrm{UO}_{2}$. The crystal structure of hypostoichiometric $\left(\mathrm{UO}_{2-x}\right)$ and hyperstoichiometric $\left(\mathrm{UO}_{2+x}\right)$ uranium dioxide has been studied extensively over the years, in particular using x-ray diffraction (XRD) [1-7]. All the studies have unanimously concluded to a contraction of the lattice constant as a function of $x$. However, at the atomic scale, the identification of the defects responsible for the lattice contraction of the fluorite structure has been a matter of debate for a long time. For instance, in the hyperstoichiometric region of the phase diagram, the shrinkage is ascribed to oxygen interstitial atoms that may cluster with different proposed geometries: the so-called 2:2:2 Willis clusters [8,9], the oxygen cuboctahedra [10], or the recently computer-discovered clusters $I_{X}^{4}[11,12]$. But the uranium vacancies cannot be excluded either [13].

To elucidate which defects are present in uranium dioxide, a point defect model could be built [14-16]. Based on formation energies obtained from first-principles calculations, the point defect model evaluates the defect concentration at thermodynamic equilibrium at infinite dilution. With these pieces of information and the volume of relaxation associated to each defect, numerical simulations can in principle calculate the lattice change as a function of the stoichiometry. However, $\mathrm{UO}_{2}$ is an insulating oxide and its defects are charged. This statement has two consequences that make the problem more complex. First of all, the point defect model has to be extended to consider all the possible charge states of the intrinsic defects and to ensure the overall charge neutrality
$[17,18]$. Second, only very recently has it been shown how to extract a meaningful volume of relaxation for charged defects out of periodic first-principles calculations [19,20]. Indeed, due to the impossibility to define an absolute electrostatic potential in periodic systems [21,22], the pressure and the stress tensor obtained from any periodic calculations are meaningless for non-neutral cells. In Ref. [20], we proposed methods to circumvent the issue and produce well-defined, transferable stresses and therefore volumes of relaxation.

In this article, we address the modeling of the latticeconstant variation of $\mathrm{UO}_{2}$ with the use of a point defect model that incorporates all the stable charge states of the intrinsic defects. The point defect model relies on first-principles calculations within the density-functional theory " $+U$ " formalism $(\mathrm{DFT}+U)[23,24]$ as is customary for correlated oxides. By combining well-defined volumes of relaxation for intrinsic defects and their equilibrium concentrations, we offer a satisfactory comparison to the available experimental data.

The article is organized as follows: In Sec. II, we review how volumes of relaxation can be obtained from periodic calculations of charged defects; in Sec. III, we describe the $\mathrm{DFT}+U$ parameters, the resulting defect formation energy, and how they are included in the point defect model; and finally, Sec. IV discusses the lattice-constant variation in the hypostoichiometric and hyperstoichiometric regimes.

\section{VOLUME OF RELAXATION OF CHARGED DEFECTS FROM FIRST PRINCIPLES}

Periodic boundary conditions make the evaluation of the electrostatic potential a mathematically ill-defined 
problem [21]. The impossibility to evaluate the electrostatic potential in quantum-mechanical calculations with periodic boundary conditions has many undesired consequences. Just to name one, the formation energy of charged systems (with an unbalanced number of electrons and protons) as evaluated from the calculations needs to be corrected [25-27].

In Ref. [22], we had clarified the role of the average electrostatic potential $\left\langle v_{H}\right\rangle$ in the total energy $E(q)$ as a function of the charge $q=Z-N$, with $Z$ being the number of protons and $N$ the number of electrons in the simulation cell. In periodic calculations, two different codes or setups may use different conventions for the average electrostatic potential, say, convention A and convention B, and we had shown [22] that the corresponding total energies are related through

$$
E^{\mathrm{A}}(q)+q\left\langle v_{H}\right\rangle_{\mathrm{A}}=E^{\mathrm{B}}(q)+q\left\langle v_{H}\right\rangle_{\mathrm{B}} .
$$

Changing code or changing pseudopotentials or projectoraugmented atomic data makes two total energy calculations impossible to compare directly. Only at $q=0$ are the energies $E^{\mathrm{A}}(0)$ and $E^{\mathrm{B}}(0)$ indeed the same.

As a consequence, the pressures, the negative first derivative of the energy with respect to the cell volume $\Omega$, calculated in the periodic approach are convention dependent,

$$
P^{\mathrm{A}}(q)-q \frac{\partial\left\langle v_{H}\right\rangle_{\mathrm{A}}}{\partial \Omega}=P^{\mathrm{B}}(q)-q \frac{\partial\left\langle v_{H}\right\rangle_{\mathrm{B}}}{\partial \Omega},
$$

and the resulting volume of relaxation is convention dependent as well. In the quadratic region around the minimum energy, the volume of relaxation, $\Delta \Omega$, is simply proportional to the pressure:

$$
\Delta \Omega^{\mathrm{A}}=\frac{P^{\mathrm{A}}}{B} \Omega,
$$

where $B$ is the bulk modulus of the host material. We have shown in a previous article [20] that the bulk modulus of the charged defective cell can be conveniently approximated by the bulk modulus of the pristine solid. The superscript A emphasizes that the obtained quantities are still convention dependent at this stage. The connection to the volume of relaxation within another convention B is easily done using Eq. (2):

$$
\begin{aligned}
\Delta \Omega^{\mathrm{B}} & =\frac{P^{\mathrm{B}}}{B} \Omega \\
& =\Delta \Omega^{\mathrm{A}}-\frac{q \Omega}{B} \frac{\partial}{\partial \Omega}\left[\left\langle v_{H}\right\rangle_{\mathrm{A}}-\left\langle v_{H}\right\rangle_{\mathrm{B}}\right] .
\end{aligned}
$$

From this last equation, we see that it is impossible to obtain the volume of relaxation for charged systems from a single periodic calculation. However, the derivative $\partial\left\langle v_{H}\right\rangle_{\mathrm{A}} / \partial \Omega$ can be connected to the absolute deformation potential [28-30] or can be made harmless by compensating with opposite charge species [20]. The latter approach does not require further interface calculations and is sufficient under the mild hypothesis of the overall charge neutrality of the macroscopic sample.

Let us exemplify how the compensation of charged defects with free holes, denoted $\mathrm{h}^{+}$, allows us to define unambiguous volumes of relaxation. It should be understood that free holes are simply a convenient charge compensator. Any other choice-free electrons (labeled $e^{-}$), self-trapped holes, charged vacancies-could play the same role. Let us consider a uranium vacancy bearing a -4 charge, $\mathrm{V}_{\mathrm{U}}^{4-}$, and let us form the volume of relaxation of these vacancies together with four free holes. These five objects are noninteracting and have to be calculated separately in independent periodic calculations. They have been chosen to form a charge-neutral group. Using Eq. (4), their total volume of relaxation reads

$$
\begin{aligned}
\Delta & \Omega^{\mathrm{B}}\left(\mathrm{V}_{\mathrm{U}}^{4-}\right)+4 \Delta \Omega^{\mathrm{B}}\left(\mathrm{h}^{+}\right) \\
= & \Delta \Omega^{\mathrm{A}}\left(\mathrm{V}_{\mathrm{U}}^{4-}\right)+4 \Delta \Omega^{\mathrm{A}}\left(\mathrm{h}^{+}\right) \\
& +4 \frac{\Omega}{B} \frac{\partial}{\partial \Omega}\left[\left\langle v_{H}\left(\mathrm{~V}_{\mathrm{U}}^{4-}\right)\right\rangle_{\mathrm{A}}-\left\langle v_{H}\left(\mathrm{~V}_{\mathrm{U}}^{4-}\right)\right\rangle_{\mathrm{B}}\right] \\
& -4 \frac{\Omega}{B} \frac{\partial}{\partial \Omega}\left[\left\langle v_{H}\left(\mathrm{~h}^{+}\right)\right\rangle_{\mathrm{A}}-\left\langle v_{H}\left(\mathrm{~h}^{+}\right)\right\rangle_{\mathrm{B}}\right],
\end{aligned}
$$

where the average electrostatic potentials of a supercell including a vacancy $\left\langle v_{H}\left(\mathrm{~V}_{\mathrm{U}}^{4-}\right)\right\rangle$ or including a free hole $\left\langle v_{H}\left(\mathrm{~h}^{+}\right)\right\rangle$have been introduced.

In Ref. [20], it has been argued that the difference between the electrostatic potential of supercells containing dilute defects vanishes to the first order. As a consequence, one can approximate the electrostatic potential averages with their bulk values:

$$
\begin{aligned}
&\left\langle v_{H}\left(\mathrm{~V}_{\mathrm{U}}^{4-}\right)\right\rangle_{\mathrm{A}} \approx\left\langle v_{H}\left(\mathrm{~h}^{+}\right)\right\rangle_{\mathrm{A}} \approx\left\langle v_{H}(\text { bulk })\right\rangle_{\mathrm{A}}, \\
&\left\langle v_{H}\left(\mathrm{~V}_{\mathrm{U}}^{4-}\right)\right\rangle_{\mathrm{B}} \approx\left\langle v_{H}\left(\mathrm{~h}^{+}\right)\right\rangle_{\mathrm{B}} \approx\left\langle v_{H}(\text { bulk })\right\rangle_{\mathrm{B}} .
\end{aligned}
$$

With these equalities correct to first order, the last two terms on the right-hand side of Eq. (5) cancel out and the announced result is obtained: The volume of relaxation of neutral groups is independent of the electrostatic convention.

We just chose to compensate the charged defects with free holes. Though arbitrary, this choice is very handy since the free hole volume of relaxation can be obtained from bulk primitive cell calculations according to

$$
\Delta \Omega^{\mathrm{A}}\left(\mathrm{h}^{+}\right)=\frac{\Omega}{B} \frac{\partial \epsilon_{\mathrm{VBM}}^{\mathrm{A}}}{\partial \Omega},
$$

where $\epsilon_{\mathrm{VBM}}^{\mathrm{A}}$ is the energy of the valence-band maximum in convention A. This last result is a consequence of the Janak theorem [31]. Its derivation is detailed in Ref. [20].

In the rest of the article, we always combine the charged defects with free holes in order to form neutral groups whose volume of relaxation is well defined and comparable with others' work. All the compensated volumes of relaxation are gathered in the Appendix. Let us insist again that the volume of relaxation of a group is obtained from distinct charged-cell calculations that have individually meaningless pressures and volumes of relaxation.

\section{INTRINSIC POINT DEFECTS OF UO}

In this section, we present the technical details of the DFT calculations and of the point defect model. We also discuss a few selected formation energies relevant for the comparison with the few available experimental data. The comprehensive list of the formation energies and of the volumes of relaxation is given in the Appendix. 


\section{A. First-principles DFT $+U$ parameters}

The first-principles calculation of the intrinsic point defects of $\mathrm{UO}_{2}$ has been the topic of numerous earlier works $[11,16,17,32-43]$. However, it is necessary to perform these calculations again here, since we are interested not only in formation energies but also in volumes of relaxation.

Our first-principles total energy and pressure calculations follow the most standard procedure for $\mathrm{UO}_{2}$. The setup is identical to the one described in Ref. [41]. We employ the VASP code [44] with projector-augmented atomic data [45]. Density-functional theory is approximated within the generalized gradient approximation $(\mathrm{GGA})+U$ exchange correlation in the formulation proposed by Liechtenstein and co-workers [23]. The on-site Coulomb repulsion $U$ and the exchange integral $J$ are set to the commonly accepted values for $\mathrm{UO}_{2}$, respectively $U=4.50 \mathrm{eV}$ and $J=0.51 \mathrm{eV}$ [46]. We take particular care with the starting density matrix as devised in the occupation matrix control technique [47] in order to mitigate the local minima issue of $\mathrm{DFT}+U$. As discussed in detail in the review by Dorado and co-authors [40], we neglect the spin-orbit coupling, since it has been constantly shown to have little effect on the energetic properties of $\mathrm{UO}_{2}$ [48-50]. We also approximate the low-temperature magnetic ordering with a collinear $1 \mathbf{k}$ antiferromagnetic order, instead of the experimental noncollinear $3 \mathbf{k}$ antiferromagnetic order. These two magnetic orderings have very close energies and are expected by all authors to yield similar formation energies for defects [40].

The defects are inserted into $2 \times 2 \times 2$ supercells based on the conventional cubic cell, resulting in 96-atom supercells. The supercell shape and volume are kept fixed to their bulk geometry with Jahn-Teller distortion. We employ a $2 \times 2 \times 2$ Monkhorst-Pack $k$-point grid. Uranium is considered to have 14 valence electrons and oxygen 6 . The plane-wave cutoff energy is set to $500 \mathrm{eV}$.

With these calculation parameters, the equilibrium crystalline structure for $\mathrm{UO}_{2}$ is an orthorhombic cell with lattice constants 5.503, 5.584, and 5.537 $\AA$. This is the so-called Jahn-Teller distorted cell. The obtained Kohn-Sham band gap is $2.41 \mathrm{eV}$.

Finally, the charged supercells need to be corrected for the spurious image interactions. Here we simply use the Madelung monopole correction [25-27] without introducing the potential alignment term, as we have verified that this term is very weak. The monopole correction uses the experimental static dielectric constant of $\mathrm{UO}_{2}, \varepsilon_{0} \approx 25$.

\section{B. Formation energies}

With this quantum-mechanical machinery, we can have access to the formation energy of each defect. The formation energy $E_{f}$ of a defect that introduces $m$ additional uraniums, $n$ additional oxygens, and $q$ positive charges in a $32-\mathrm{UO}_{2}-$ formula supercell is defined as

$$
\begin{aligned}
E_{f}^{m, n, q}\left(\mu_{e}\right)= & E\left(\mathrm{U}_{32+m} \mathrm{O}_{64+n}\right) \\
& -\frac{32+m}{32} E\left(\mathrm{U}_{32} \mathrm{O}_{64}\right)-\frac{n-2 m}{2} E\left(\mathrm{O}_{2}\right) \\
& +q\left(\epsilon_{\mathrm{VBM}}+\mu_{e}\right)+\Delta E_{\text {Madelung }} .
\end{aligned}
$$

The energies are referred to the oxygen molecule in its triplet ground state $E\left(\mathrm{O}_{2}\right)$ within the Perdew-Burke-Ernzerhof GGAPBE [51]: these are the so-called oxygen-rich conditions. We chose to refer all the formation energies to this well-defined reference. This makes our values easily comparable with future works (see the Appendix). Note that the precise value of the oxygen chemical potential does not matter for the properties we are interested in here. The formation energy is a function of the electron chemical potential $\mu_{e}$ considered as an external parameter at this stage.

In this study, we have considered the most comprehensive list of intrinsic defects in $\mathrm{UO}_{2}$ known today. First, we have calculated the hole polaron, i.e., a $\mathrm{U}^{5+}$ ion in $\mathrm{UO}_{2}$, and the electron polaron, a $\mathrm{U}^{3+}$ ion. Second, we have considered the uranium defects: the uranium vacancy and the uranium self-interstitial in an octahedral site. Third, the simple oxygen defects, oxygen vacancy and oxygen self-interstitial in an octahedral site, have been evaluated. Fourth, we have calculated the oxygen clusters described by Andersson and co-authors [11]: the two-oxygen interstitials $\mathrm{I}_{\mathrm{X}}^{2}$ and the four-oxygen interstitials $\mathrm{I}_{\mathrm{X}}^{4}$. And finally, we have evaluated the five-oxygen interstitial cluster in cuboctahedral shape, $\mathrm{I}_{\mathrm{C}}^{5}$, which is the building block for the $\mathrm{U}_{4} \mathrm{O}_{9}$ crystalline structure [10].

We obtained formation energies in line with Ref. [41], with which we share a similar technical setup. All the formation energies are given in the Appendix. Let us just describe here a few selected formation energies.

Figure 1 presents the formation energy for the stoichiometric groups: the Schottky defect, one isolated uranium vacancy and two isolated oxygen vacancies, the uranium Frenkel pair, one isolated uranium self-interstitial and one isolated uranium vacancy, and the oxygen Frenkel pair, one isolated oxygen selfinterstitial and one isolated oxygen vacancy. These energies do

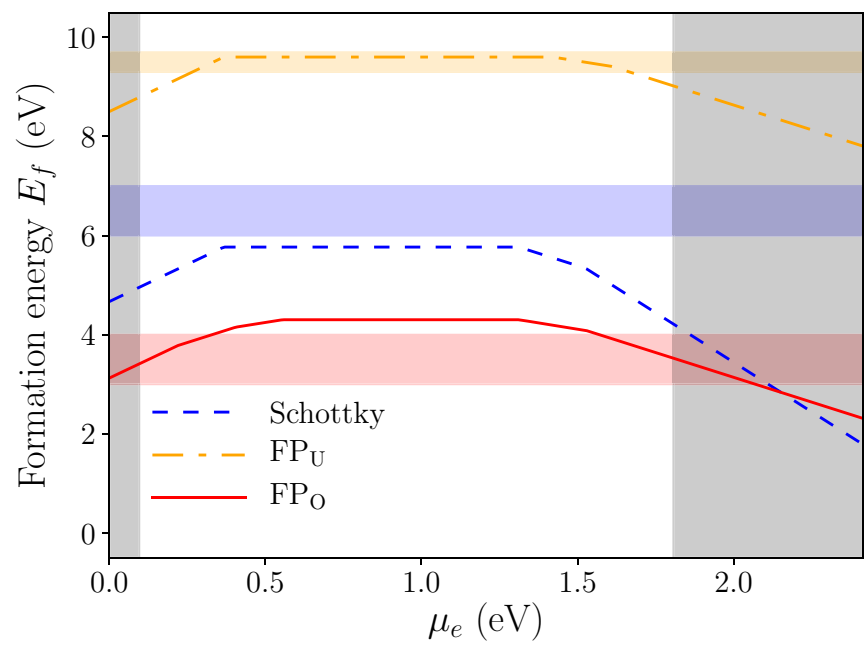

FIG. 1. Formation energies (eV) of the Schottky trio (dashed blue line), of the uranium Frenkel pair (dash-dotted orange line), and of the oxygen Frenkel pair (solid red line) as a function of the electron chemical potential $\mu_{e}$ in $\mathrm{eV}$. The electron chemical potential is referred to the valence-band maximum energy of bulk $\mathrm{UO}_{2}$. However, due to the formation of hole and electron polarons, the actual range for $\mu_{e}$ is limited and cannot enter the gray shaded areas in the dilute limit. The experimental estimates from Ref. [15] are symbolized by the horizontal colored areas. 


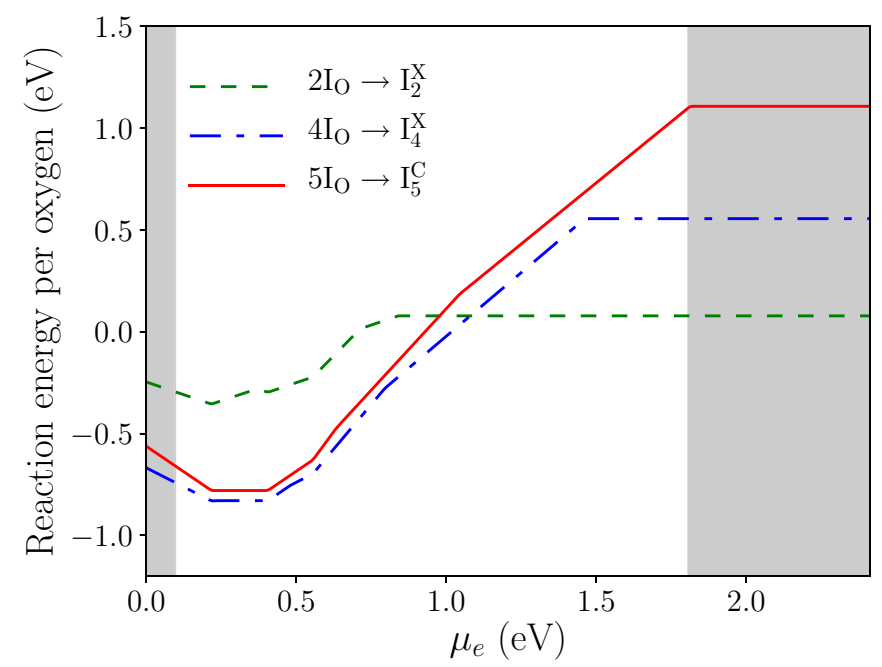

FIG. 2. Reaction energies per extra oxygen atom $(\mathrm{eV})$ for the $\mathrm{I}_{\mathrm{X}}^{2}$ (dashed green line), the $\mathrm{I}_{\mathrm{X}}^{4}$ (dash-dotted blue line), and the $\mathrm{I}_{\mathrm{C}}^{5}$ oxygen (solid red line) clusters as a function of the electron chemical potential $\mu_{e}$ in $\mathrm{eV}$. The electron chemical potential is referred to the valenceband maximum energy of bulk $\mathrm{UO}_{2}$. However, due to the formation of hole and electron polarons, the actual range for $\mu_{e}$ is limited and cannot enter the gray shaded areas in the dilute limit.

not depend on the oxygen chemical potential but are a function of the electron chemical potential $\mu_{e}$. The reachable values for $\mu_{e}$ are bounded by the formation of isolated $\mathrm{U}^{5+}$ and $\mathrm{U}^{3+}$ ions. The energies can be compared to the experimental estimates obtained from self-diffusion measurements as summarized by Matzke [15]. The agreement with respect to experiment is good even though the experimental values have no dependence on $\mu_{e}$.

Most noticeably, we observe, in agreement with our previous studies $[16,17]$, that the formation energy of uranium vacancies is very low and that this defect is likely to be dominant for hyperstoichiometric $\mathrm{UO}_{2+x}$. Unfortunately, $\mathrm{UO}_{2+x}$ is usually thought as being devoid of uranium vacancies from density measurements [2,52] and from neutron diffraction [8,53], which is sensitive to oxygen interstitials. This question is addressed in detail in the discussion in Sec. IV B.

Also relevant to the hyperstoichiometric regime are the reaction energies of oxygen self-interstitials when they form clusters. In Fig. 2, we plot these energies as a function of the electron chemical potential $\mu_{e}$. In the lower part of the band gap, typical conditions for $\mathrm{UO}_{2+x}$, the oxygen clusters are strongly bound with negative reaction energies. It appears that the $\mathrm{I}_{\mathrm{X}}^{2}$ clusters are much less favorable than the $\mathrm{I}_{\mathrm{X}}^{4}$ and $\mathrm{I}_{\mathrm{C}}^{5}$ clusters and that $\mathrm{I}_{\mathrm{X}}^{4}$ is indeed the most stable oxygen cluster in agreement with Andersson and co-workers [12]. These energies are independent of the oxygen chemical potential and can be easily compared to other theoretical studies.

With these calculated formation energies, we can feed the point defect model built to evaluate the equilibrium defect concentrations.

\section{Point defect model}

First introduced by Matzke [15] and Lidiard [14], the point defect model in $\mathrm{UO}_{2}$ allows one to calculate the concentration of the different point defects assuming thermodynamic equilibrium with an $\mathrm{O}_{2}$ atmosphere at a given temperature and a given partial pressure and assuming the point defects have no interactions (dilute limit). Here we extend the regular point defect model to oxygen clusters considered as point defects themselves and to charged defects following Ref. [17]. This is, to the best of our knowledge, the most comprehensive point defect model built for $\mathrm{UO}_{2}$ as of today.

Let us exemplify the use of the law of mass action for one particular defect, say, $\mathrm{I}_{\mathrm{X}}^{4}$ with charge state $q$. The formation chemical reaction reads

$$
\varnothing+2 \mathrm{O}_{2} \rightarrow \mathrm{I}_{\mathrm{X}}^{4 q}+q e^{-},
$$

and the corresponding law of mass action states

$$
\left[\mathrm{I}_{\mathrm{X}}^{4 q}\right]=3\left(P_{\mathrm{O}_{2}}\right)^{2} \exp \left[-E_{f}^{0,4, q}\left(\mu_{e}\right) / k_{B} T\right],
$$

where the formation energy defined in Eq. (8) has been introduced. In this work, the notation for concentrations with brackets [.] refers to a concentration per $\mathrm{UO}_{2}$ formula unit. The prefactor 3 accounts for the three possible configurations of an $\mathrm{I}_{\mathrm{X}}^{4}$ cluster for each formula unit. Note that when stating Eq. (10) in terms of formation energy and not formation free energy, we neglect all the sources of entropy besides the configurational one. In particular, all the point defect models we are aware of for $\mathrm{UO}_{2}$ neglect the vibrational entropy of the defects. Going beyond this approximation is nowadays way beyond the reach of the first-principles DFT $+U$ method.

Equations similar to Eq. (10) can be written for each defect type $\mathrm{X}^{q}$ (polarons, vacancies, self-interstitials, and clusters). The only remaining unknown is the electron chemical potential $\mu_{e}$ that can be determined by imposing the overall charge neutrality of the $\mathrm{UO}_{2}$ sample:

$$
\sum_{\mathrm{X}^{q}} q\left[\mathrm{X}^{q}\right]+\left[\mathrm{h}^{+}\right]-\left[e^{-}\right]=0,
$$

with $\left[\mathrm{h}^{+}\right]$and $\left[e^{-}\right]$being the concentrations (per formula unit) of the delocalized valence holes and the delocalized conduction electrons, respectively [54]. These two last quantities only depend on the bulk density of states, on the imposed temperature, and on $\mu_{e}$.

Finally, the stoichiometry $x$ is calculated by accounting for the oxygen and uranium change introduced by each defect type weighted by its actual concentration. The same approach gives access to the overall volume change of a formula unit $\Delta \Omega$ :

$$
\Delta \Omega=\sum_{\mathrm{X}^{q}} \Delta \Omega\left(\mathrm{X}^{q}\right)\left[\mathrm{X}^{q}\right]+\Delta \Omega\left(e^{-}\right)\left[e^{-}\right]+\Delta \Omega\left(\mathrm{h}^{+}\right)\left[\mathrm{h}^{+}\right] .
$$

The volumes of relaxation introduced in Eq. (12) are the absolute volumes of relaxation that we would like to avoid calculating. Following Sec. II, let us subtract from Eq. (12) the volume of relaxation of the free holes times the left-hand side of Eq. (11). This does not affect the total volume change thanks to electroneutrality and we obtain our working equation:

$$
\begin{aligned}
\Delta \Omega= & \sum_{\mathrm{X}^{q}}\left\{\Delta \Omega\left(\mathrm{X}^{q}\right)-q \Delta \Omega\left(\mathrm{h}^{+}\right)\right\}\left[\mathrm{X}^{q}\right] \\
& +\left\{\Delta \Omega\left(e^{-}\right)+\Delta \Omega\left(\mathrm{h}^{+}\right)\right\}\left[e^{-}\right] .
\end{aligned}
$$




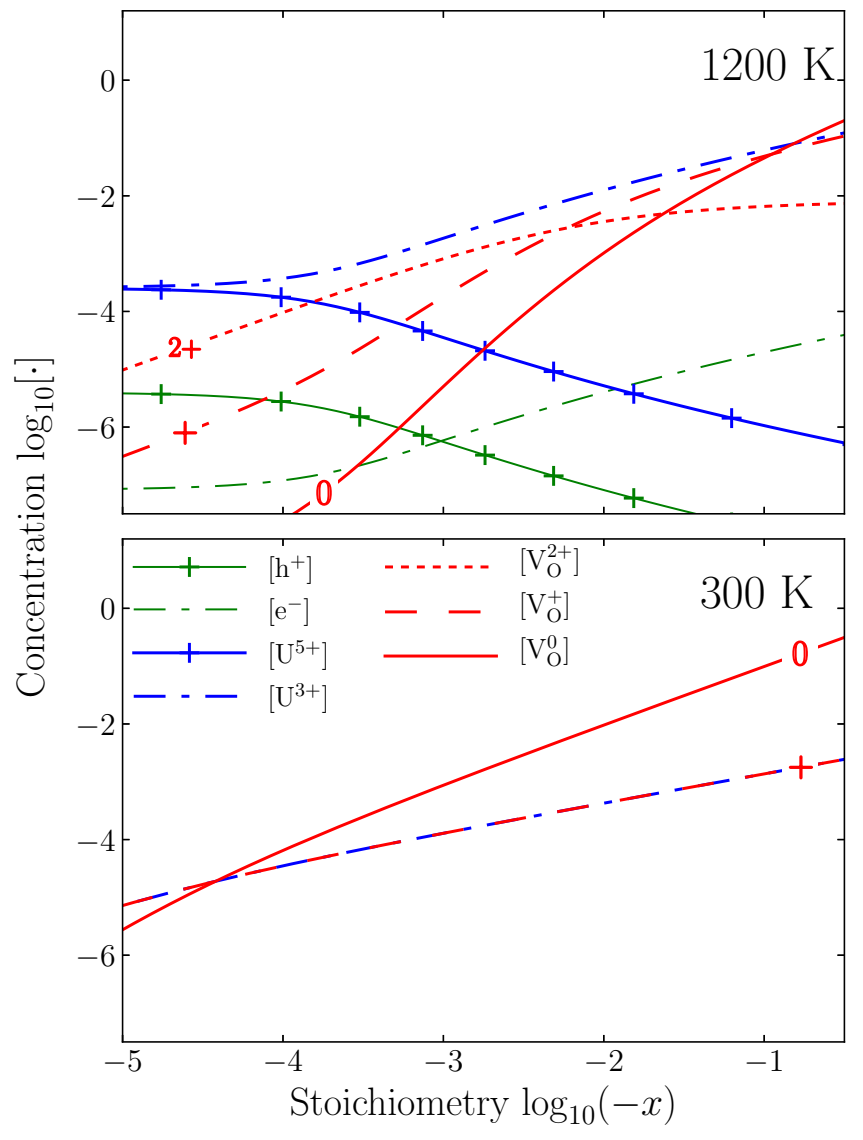

FIG. 3. Concentration per formula unit of the different species as a function of the stoichiometry of $\mathrm{UO}_{2+x}$ for negative $x$ (hypostoichiometric regime) at $1200 \mathrm{~K}$ (upper panel) and at $300 \mathrm{~K}$ (lower panel). Only the dominant species are reported: delocalized holes and electrons (thin green lines), localized polarons (thick blue lines), and oxygen vacancies for different charge states (red lines).

The volumes of relaxation grouped in each curly bracket are independent of the electrostatic potential convention as we desired. Note that the choice of compensating free holes is completely arbitrary. Finally, the relative change of lattice constant will be obtained as one-third of the relative change of the cell volume.

With this, we are now able to address the first-principles calculation of the lattice-constant variation.

\section{LATTICE-CONSTANT CHANGE IN NONSTOICHIOMETRIC $\mathrm{UO}_{2}$}

\section{A. Hypostoichiometric regime}

In the hypostoichiometric regime, the measurements of the lattice-constant variation are quite scarce. This statement owes much to the challenge of synthesizing good quality samples with no metallic uranium. In Ref. [1], Kapshukov and co-workers proposed a route to reach hypostoichiometry through the reduction of $\mathrm{UO}_{2}$ at relatively high temperature $(1200 \mathrm{~K})$. Then they quenched their sample and performed XRD determination of the lattice constant at room temperature. It is not clear at this stage which temperature is most relevant (the synthesis temperature or the analysis temperature). We applied our point defect model for the two temperatures then.

The calculated defect concentrations are reported in Fig. 3 as a function of the stoichiometry at $1200 \mathrm{~K}$ (upper panel) and at $300 \mathrm{~K}$ (lower panel). First of all, whatever the temperature, among all the defects considered in the model, only the localized polarons and the oxygen vacancies are present with non-negligible concentrations. Delocalized charge carriers have much lower concentrations. However, the prevailing charge state of the oxygen vacancies is strongly dependent on the temperature. At high temperature, the main occurring defect is the $2+$ oxygen vacancy in the intrinsic regime, $\log _{10}(-x) \approx-4$. The charge compensation is ensured by a slightly unbalanced proportion of the positive and negative polarons. For stoichiometries comprised in between $\mathrm{UO}_{1.993}$ and $\mathrm{UO}_{1.89}$, the $1+$ oxygen vacancies are prevailing. Their charge is almost fully compensated by negative polarons, $\mathrm{U}^{3+}$. The neutral vacancies become dominant only for very strong departure from stoichiometry $(x<-0.11)$. The importance of the singly positively charged oxygen vacancies at high temperature had already been emphasized in Ref. [17].

At room temperature, the intrinsic regime is very narrow as very few charges (be they delocalized or localized) are thermally generated. Already with a stoichiometry as low as $\log _{10}(-x)>-4.5$, the sample becomes dominated by neutral vacancies. The negative polarons $\mathrm{U}^{3+}$ precisely compensate the rarely occurring $\mathrm{V}_{\mathrm{O}}^{+}$. Note that most point defect models available in the literature only consider $\mathrm{V}_{\mathrm{O}}^{2+}$, which would miss the physics we just described.

Let us now report in Fig. 4 the lattice-constant variation for the two considered temperatures. Our data are compared to the measurements of Ref. [1]. The agreement between the XRD lattice constant and our model at room temperature is strikingly good. The dependence of the lattice constant with respect to $x$ is almost linear according to the point defect model and can be modeled by the relation

$$
a(x) \approx a_{0}-0.263 x \AA,
$$

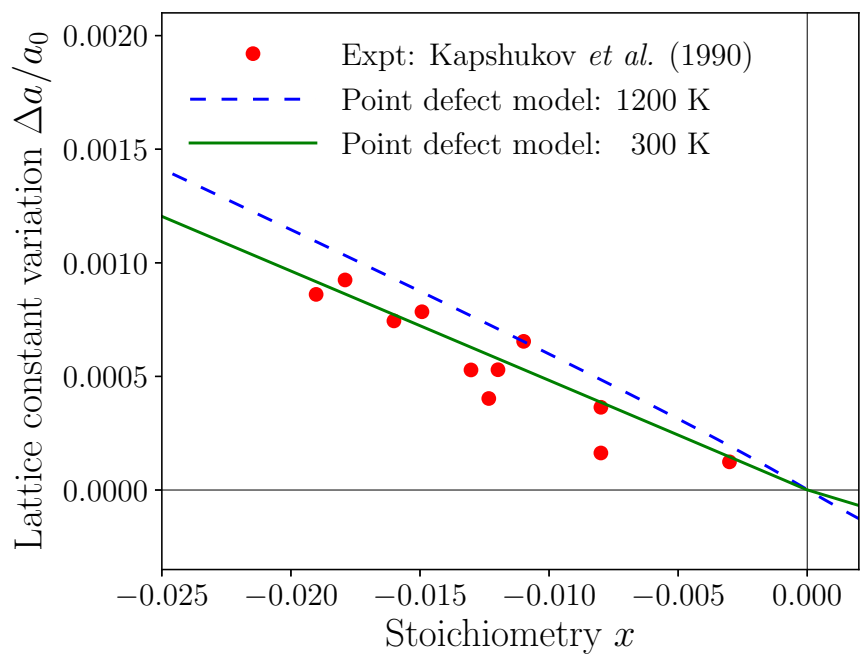

FIG. 4. Relative change in lattice constant as a function of $x$ in $\mathrm{UO}_{2+x}$ for negative $x$ (hypostoichiometric regime). The point defect model at $1200 \mathrm{~K}$ (dashed blue line) and at $300 \mathrm{~K}$ (solid green line) is compared to the XRD data from Ref. [1] (red circles). 
TABLE I. GGA $+U$ formation energies $E_{f}$ and volume of relaxation $\Delta \Omega$ for all the charged defects considered in the point defect model.

\begin{tabular}{|c|c|c|c|}
\hline Defect & Charge, $q$ & $\begin{array}{c}E_{f} \\
(\mathrm{eV})\end{array}$ & $\begin{array}{c}\Delta \Omega-q \Delta \Omega\left(\mathrm{h}^{+}\right) \\
\left(\AA^{3}\right)\end{array}$ \\
\hline $\mathrm{h}^{+}$ & +1 & 0.000 & 0.00 \\
\hline$e^{-}$ & -1 & 2.414 & -0.44 \\
\hline $\mathrm{U}^{5+}$ & +1 & -0.096 & 0.52 \\
\hline $\mathrm{U}^{3+}$ & -1 & 1.807 & 0.73 \\
\hline $\mathrm{V}_{\mathrm{O}}$ & +2 & 2.544 & 7.57 \\
\hline $\mathrm{V}_{\mathrm{O}}$ & +1 & 3.856 & 6.27 \\
\hline $\mathrm{V}_{\mathrm{O}}$ & 0 & 5.382 & 6.13 \\
\hline $\mathrm{I}_{\mathrm{O}}$ & +1 & 0.581 & -3.26 \\
\hline $\mathrm{I}_{\mathrm{O}}$ & 0 & 0.798 & -3.80 \\
\hline $\mathrm{I}_{\mathrm{O}}$ & -1 & 1.203 & -0.26 \\
\hline $\mathrm{I}_{\mathrm{O}}$ & -2 & 1.758 & 0.77 \\
\hline $\mathrm{I}_{\mathrm{X}}^{2}$ & +1 & 0.668 & 0.28 \\
\hline $\mathrm{I}_{\mathrm{X}}^{2}$ & 0 & 1.191 & -0.09 \\
\hline $\mathrm{I}_{\mathrm{X}}^{2}$ & -1 & 1.406 & 0.72 \\
\hline $\mathrm{I}_{\mathrm{X}}^{2}$ & -2 & 2.208 & 2.09 \\
\hline $\mathrm{I}_{\mathrm{X}}^{2}$ & -3 & 2.832 & 2.49 \\
\hline $\mathrm{I}_{\mathrm{X}}^{2}$ & -4 & 3.672 & 2.58 \\
\hline $\mathrm{I}_{\mathrm{X}}^{4}$ & +1 & -0.351 & -2.82 \\
\hline $\mathrm{I}_{\mathrm{X}}^{4}$ & 0 & -0.127 & -4.29 \\
\hline $\mathrm{I}_{\mathrm{X}}^{4}$ & -1 & 0.355 & -4.67 \\
\hline $\mathrm{I}_{\mathrm{X}}^{4}$ & -2 & 1.210 & -4.49 \\
\hline $\mathrm{I}_{\mathrm{X}}^{4}$ & -3 & 1.945 & -3.28 \\
\hline $\mathrm{I}_{\mathrm{X}}^{4}$ & -4 & 3.487 & -3.92 \\
\hline $\mathrm{I}_{\mathrm{X}}^{4}$ & -5 & 4.921 & -2.98 \\
\hline $\mathrm{I}_{\mathrm{X}}^{4}$ & -6 & 6.707 & -2.13 \\
\hline $\mathrm{I}_{\mathrm{X}}^{4}$ & -7 & 7.897 & -2.94 \\
\hline $\mathrm{I}_{\mathrm{X}}^{4}$ & -8 & 9.254 & -2.67 \\
\hline $\mathrm{I}_{\mathrm{C}}^{5}$ & 0 & 0.090 & -0.57 \\
\hline $\mathrm{I}_{\mathrm{C}}^{5}$ & -1 & 0.900 & -0.49 \\
\hline $\mathrm{I}_{\mathrm{C}}^{5}$ & -2 & 1.358 & 0.18 \\
\hline $\mathrm{I}_{\mathrm{C}}^{5}$ & -3 & 2.404 & -1.89 \\
\hline $\mathrm{I}_{\mathrm{C}}^{5}$ & -4 & 3.447 & -2.31 \\
\hline $\mathrm{I}_{\mathrm{U}}$ & +4 & 8.922 & 39.18 \\
\hline $\mathrm{I}_{\mathrm{U}}$ & +3 & 10.343 & 36.51 \\
\hline $\mathrm{I}_{\mathrm{U}}$ & +2 & 11.954 & 35.58 \\
\hline $\mathrm{V}_{\mathrm{U}}$ & -1 & -0.420 & -9.24 \\
\hline $\mathrm{V}_{\mathrm{U}}$ & -2 & 0.084 & -9.89 \\
\hline $\mathrm{V}_{\mathrm{U}}$ & -3 & 0.322 & -9.74 \\
\hline $\mathrm{V}_{\mathrm{U}}$ & -4 & 0.680 & -18.56 \\
\hline
\end{tabular}

which matches nicely the slope coefficient reported by Kapshukov et al., -0.255 [1].

Calculations and experiment both agree on the dilatation of the lattice with $x$. The lattice-constant dilation is almost entirely due to the oxygen vacancies. It is quite remarkable that the oxygen vacancies have a large positive volume of relaxation. Intuitively, one would have expected that removing atoms from $\mathrm{UO}_{2}$ would induce a shrinkage of the structure. This is the case, for instance, for the uranium vacancies (see Table I). Let us propose that in the absence of a small oxygen atom the largely charged uranium ions repel each other strongly.
Concerning the experimental interpretation, we conclude that the oxygen vacancies are generated during the hightemperature synthesis. Then during the quenching, the oxygen vacancy concentration remains constant; however, the charge equilibration is given enough time to happen. The vacancies $\mathrm{V}_{\mathrm{O}}^{+}$and polarons $\mathrm{U}^{3+}$ are then converted into neutral vacancies $\mathrm{V}_{\mathrm{O}}^{0}$. The lattice constant is then modified as shown in Fig. 4, since the volume of relaxation of $\mathrm{V}_{\mathrm{O}}^{0}$ is around $6 \AA^{3}$, whereas the combined volume of relaxation of $\mathrm{V}_{\mathrm{O}}^{1+}$ and $\mathrm{U}^{3+}$ is somewhat larger $\left(\sim 7 \AA^{3}\right)$.

The outstanding agreement with experimental latticeconstant variation is a strong support to the predictive power of the point defect model based on DFT $+U$ data.

\section{B. Hyperstoichiometric regime}

For hyperstoichiometric $\mathrm{UO}_{2}$, there are many experimental determinations of the lattice constant in the literature [2-7]. At low temperature, the range of stability of the solid solution $\mathrm{UO}_{2+x}$ is very narrow because the $\mathrm{U}_{4} \mathrm{O}_{9}$ crystalline phase forms [55]. In the following, we then focus on hightemperature experiments only $(\sim 1400 \mathrm{~K})$. At these temperatures, the $\mathrm{UO}_{2+x}$ stoichiometry can achieve $x=0.23$ without turning into the $\mathrm{U}_{4} \mathrm{O}_{9}$ phase, which allows us to employ our point defect model of $\mathrm{UO}_{2}$. The experimental XRD data we refer to in the following were either measured directly at high temperature $[2,4]$ or measured at room temperature but with a rapid quench after the high-temperature synthesis [3].

The upper panel of Fig. 5 shows the species concentrations as a function of the stoichiometry $x$ as obtained from the point defect model at $1400 \mathrm{~K}$. For the sake of clarity, only the total concentration for each defect type is reported: the competing charge states have been summed up. As stated above in Sec. III, the uranium vacancies have a very low formation energy in DFT $+U$ and it is not a surprise that it is predicted to be the dominating defect whatever the stoichiometry. However, as mentioned earlier, there is a consensus that the oxidation of $\mathrm{UO}_{2}$ is mediated through the insertion of oxygen interstitial atoms, not through the formation of uranium vacancies. But the clues are only indirect:

(i) From neutron diffraction [8,53], $\mathrm{UO}_{2+x}$ is known to incorporate oxygen interstitials. This is of course not a proof that uranium vacancies are absent.

(ii) From density measurements [2,52], $\mathrm{U}_{4} \mathrm{O}_{9}$ has been proven to have a higher density than $\mathrm{UO}_{2}$, therefore ruling out the creation of uranium vacancies in $\mathrm{U}_{4} \mathrm{O}_{9}$.

Again these measurements were performed on $\mathrm{U}_{4} \mathrm{O}_{9}$ and not on a direct observation of $\mathrm{UO}_{2+x}$. The only experimental work concluding with the occurrence of some uranium vacancies that we are aware of is Ref. [13]. The discrepancy between the calculated stability of uranium vacancies and their absence in the actual samples, the "uranium vacancy problem," had been already mentioned in the past [16]. It appears to be a common feature of all the first-principles simulations of point defects in uranium dioxide.

The consequence of incorporating uranium vacancies is better appraised from their role in the lattice constant, as plotted in Fig. 6. There, the lattice constant is shown to shrink much more in the point defect model than in the experimental 


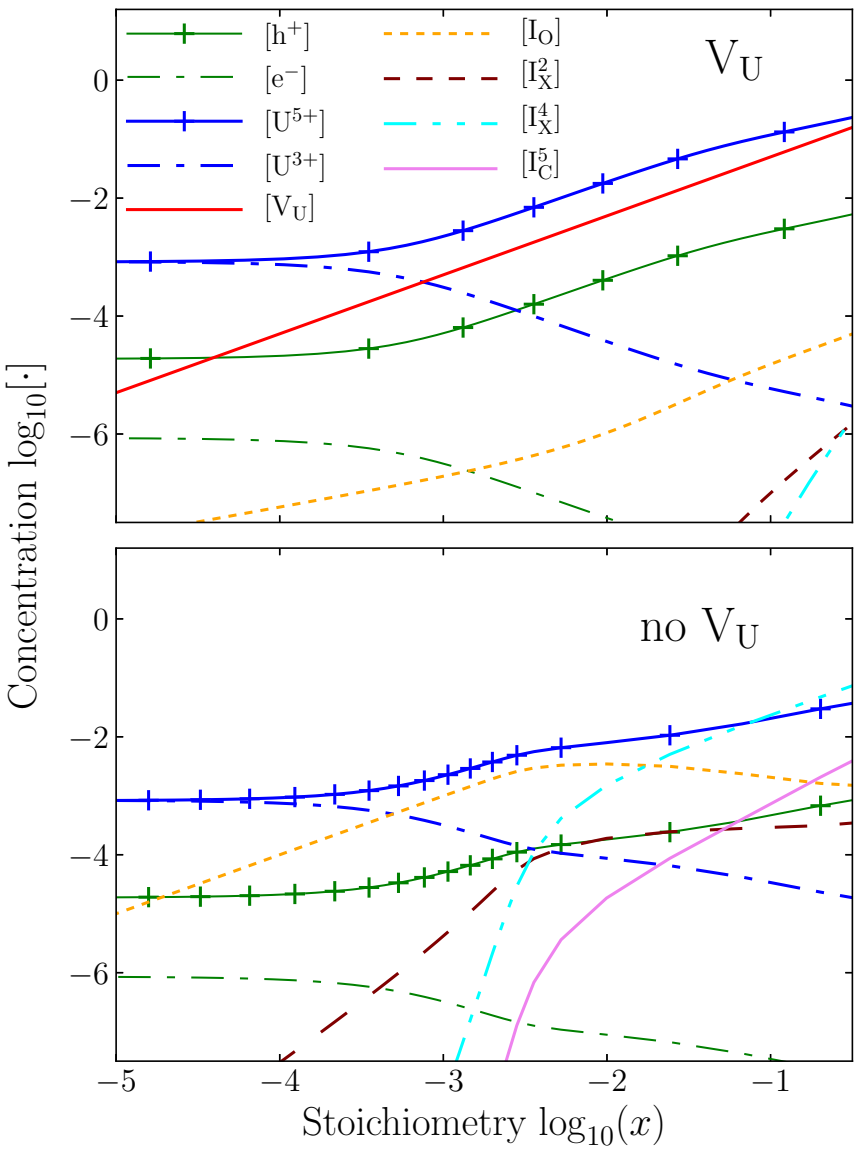

FIG. 5. Concentration per formula unit of the different species as a function of the stoichiometry of $\mathrm{UO}_{2+x}$ for positive $x$ (hyperstoichiometric regime) at $1400 \mathrm{~K}$ including (upper panel) or not (lower panel) uranium vacancies. Only the dominant species are reported: delocalized holes and electrons (thin green lines), localized polarons (thick blue lines), uranium vacancies (solid red line), oxygen interstitials (dashed orange line), and oxygen clusters (short dashed brown line for $\mathrm{I}_{\mathrm{X}}^{2}$, dashed-double-dotted cyan line for $\mathrm{I}_{\mathrm{X}}^{4}$, and dashedtriple-dotted pink line for $\mathrm{I}_{\mathrm{C}}^{5}$ ).

measurements. Indeed, among the calculated defects (see the Appendix), the uranium vacancies have the most negative volume of relaxation.

We would like here to follow a speculative path: Let us assume, as experiments encourage us to do, that, for some reason, the uranium vacancies are not present in uranium dioxide. We can simply disregard them in the point defect model. The population of defects then calculated is reported in the lower panel of Fig. 5. The isolated oxygen self-interstitial dominates up to $x=0.01$, and then the very stable oxygen cluster $\mathrm{I}_{\mathrm{X}}^{4}$ prevails. As a consequence, the dependence of the lattice constant as a function of $x$ is much weaker as shown in Fig. 6 and as fitted through

$$
a(x) \approx a_{0}-0.046 x \AA
$$

This is in very good agreement with Dodé and Touzelin's slope of -0.059 [4] and in reasonable agreement with Lynds and co-workers' value of -0.094 [3].

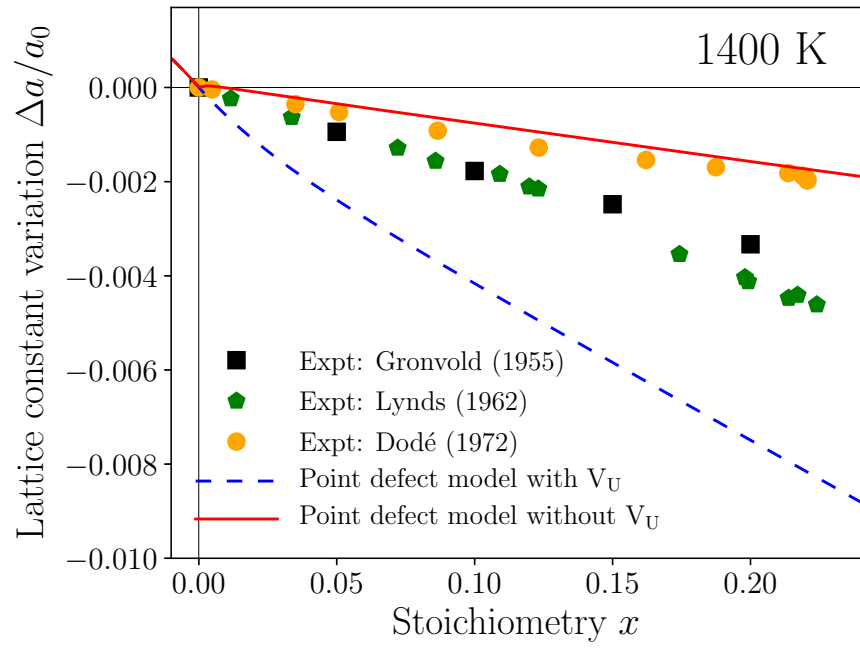

FIG. 6. Relative change in lattice constant as a function of $x$ in $\mathrm{UO}_{2+x}$ for positive $x$ (hyperstoichiometric regime) at $1400 \mathrm{~K}$. The point defect models (lines) are compared to the high-temperature XRD data (symbols).

The comparison with respect to experiment therefore supports the assumption that the $\mathrm{UO}_{2+x}$ samples are indeed devoid of uranium vacancies.

We can propose an explanation for the absence of uranium vacancies in oxidized $\mathrm{UO}_{2}$ even though these defects are predicted to be thermodynamically dominant. The formation of uranium vacancies inside the sample requires uranium cation transport from the sample to its surfaces. As reviewed by Matzke in Ref. [15], the self-diffusion of uranium in $\mathrm{UO}_{2}$ and $\mathrm{UO}_{2+x}$ is extremely slow-orders of magnitude slower than the self-diffusion of oxygen. Matzke proposes an Arrhenius fit to represent the diffusion coefficient for stoichiometric $\mathrm{UO}_{2}$ with an activation energy of $5.6 \mathrm{eV}$. A more recent review and data analysis by Moore and co-authors [56] proposes similar fits for $D_{\mathrm{UO}_{2+x}}^{U}$, however, extended to the hyperstoichiometric regime:

$$
\begin{aligned}
D_{\mathrm{UO}_{2}}^{\mathrm{U}} & =0.171 \exp \left(-5.30 \mathrm{eV} / k_{B} T\right) \mathrm{cm}^{2} / \mathrm{s}, \\
D_{\mathrm{UO}_{2.1}}^{\mathrm{U}} & =0.078 \exp \left(-4.09 \mathrm{eV} / k_{B} T\right) \mathrm{cm}^{2} / \mathrm{s} .
\end{aligned}
$$

These diffusion activation energies are in line with the range of values derived from first principles in Ref. [57].

Let us exemplify their consequence on a macroscopic scale. Consider a Gaussian solution to the one-dimensional diffusion equation and find how much time is required to experience a $0.1 \%$ change of uranium concentration $1 \mathrm{~mm}$ away from the distribution center. A change of $0.1 \%$ in concentration is a realistic target to account for hyperstoichiometric $\mathrm{UO}_{2+x}$ with $x=0.01$. One millimeter is a reasonable length scale for a small macroscopic $\mathrm{UO}_{2}$ sample. Even at an elevated temperature around $2000 \mathrm{~K}$, though measurable on the length scale of diffusion experiments $(\sim 0.5 \mu \mathrm{m})$ [58,59], the uranium self-diffusion coefficient is still very low: $D_{\mathrm{UO}_{21}}^{\mathrm{U}}=3.8 \times$ $10^{12} \mathrm{~cm}^{2} / \mathrm{s}$. Hence, the concentration $1 \mathrm{~mm}$ away would start to change with a $0.1 \%$ magnitude after a time scale of about a year. At a lower temperature around $1000 \mathrm{~K}$, the 
age of the earth [60] would be still too short to observe any change! As a consequence, we conclude that the formation of uranium vacancies is diffusion limited. It is impossible that the uranium sublattice reaches thermodynamic equilibrium in a macroscopic sample and it is not a contradiction that the uranium vacancies have a very low formation energy in the first-principles calculations, and yet they are not observed in the actually synthesized samples.

The $\mathrm{UO}_{2}$ grown samples are then better accounted for with a point defect model that does not consider uranium vacancies. This is confirmed by the lattice variation observed in Fig. 6.

\section{CONCLUSION}

In this article, on the one hand, we have developed a new point defect model for uranium dioxide based on firstprinciples GGA $+U$ calculations. This point defect model considers an unprecedented variety of species: it contains electron and hole polarons, the intrinsic point defects, and the stable oxygen interstitial clusters. For all these objects, all their relevant charge states have been incorporated. This point defect model has allowed us to evaluate the equilibrium concentrations for both hypo- and hyperstoichiometric $\mathrm{UO}_{2}$.

On the other hand, we have evaluated the volumes of relaxation of all the defects for all their significant charge states. This task relies on the recent theoretical advances reported in Ref. [20]. Weighting the volumes of relaxation of the defects with their equilibrium concentrations yields the lattice contraction or dilation as a function of the stoichiometry $x$ in $\mathrm{UO}_{2+x}$.

With the point defect models and the volume of relaxation, we have been able to nicely reproduce the experimental lattice-constant variation. In the hypostoichiometric regime, the $\mathrm{UO}_{2}$ stoichiometry is governed by the oxygen vacancies, which become charge neutral at room temperature. The oxygen vacancies present a large and positive volume of relaxation, which is quite unexpected.

In the hyperstoichiometric regime, the lattice variation is clearly incompatible with the presence of uranium vacancies as predicted by the complete point defect model, which assumes thermodynamical equilibrium. Based on experimental diffusion data, we argue that the transport of uranium cations is extremely slow and, thus, that the concentration of uranium vacancies in the actual samples is diffusion limited and cannot reach the thermodynamic equilibrium on the experimental time scale. When discarding $\mathrm{V}_{\mathrm{U}}$ from the point defect model, the predicted lattice constant as a function of stoichiometry varies in rather good agreement with experiment.

The machinery presented here is by no means limited to uranium dioxide. It could be transposed to address the lattice variation of other nonstoichiometric semiconducting crystals.

\section{ACKNOWLEDGMENTS}

We are grateful to L. Desgranges for illuminating insights on experimental aspects. This work was performed using HPC resources from GENCI-CCRT-TGCC (Grant No. $2017-$ 096018).

\section{APPENDIX: FORMATION ENERGIES AND VOLUMES OF RELAXATION USED IN THE POINT DEFECT MODEL}

In Table I, we provide the formation energies evaluated according to Eq. (8) for an electron chemical potential $\mu_{e}$ set at the valence-band maximum of bulk $\mathrm{UO}_{2}$, for an oxygen chemical potential $\mu_{\mathrm{O}}$ set at half the $\mathrm{O}_{2}$ molecule energy (within the GGA-PBE in the triplet ground state), and the uranium chemical potential $\mu_{\mathrm{U}}$ imposed by $\mathrm{UO}_{2}$ and $\mathrm{O}_{2}$, $\mu_{\mathrm{U}}=E\left(\mathrm{UO}_{2}\right)-E\left(\mathrm{O}_{2}\right)$ (oxygen-rich conditions).

The volumes of relaxation are given for charge-neutral groups using compensating delocalized holes as explained in Sec. II. In practice, the volumes reported in Table I have been obtained through

$$
\Delta \Omega\left(\mathrm{X}^{q}\right)-q \Delta \Omega\left(\mathrm{h}^{+}\right)=\frac{\Omega}{B}\left[P^{\mathrm{A}}\left(\mathrm{X}^{q}\right)-q \frac{\partial \epsilon_{\mathrm{VBM}}^{\mathrm{A}}}{\partial \Omega}\right],
$$

where $P^{\mathrm{A}}\left(\mathrm{X}^{q}\right)$ is the pressure obtained at the end of a fixed supercell calculation containing the defect $\mathrm{X}^{q}$. The convention dependence of $P$ and of $\epsilon_{\mathrm{VBM}}$ have been emphasized through the use of superscript A.
[1] I. I. Kapshukov, N. V. Lyalyushkin, L. V. Sudakov, A. S. Bevz, and O. V. Skiba, J. Radioanal. Nucl. Chem. 143, 213 (1990).

[2] F. Grønvold, J. Inorg. Nucl. Chem. 1, 357 (1955).

[3] L. Lynds, W. A. Young, J. S. Mohl, and G. G. Libowitz, Adv. Chem. 39, 58 (1963).

[4] M. Dodé and B. Touzelin, Rev. Chim. Miner. 9, 139 (1972).

[5] V. A. Alekseyev, L. A. Anan'yeva, and R. P. Rafal'skiy, Int. Geol. Rev. 23, 1229 (1981).

[6] K. Teske, H. Ullmann, and D. Rettig, J. Nucl. Mater. 116, 260 (1983).

[7] J. M. Elorrieta, L. J. Bonales, N. Rodriguez-Villagra, V. G. Baonza, and J. Cobos, Phys. Chem. Chem. Phys. 18, 28209 (2016).

[8] B. T. M. Willis, Acta Crystallogr. Sect. A 34, 88 (1978).
[9] G. C. Allen, P. A. Tempest, and J. W. Tyler, Nature (London) 295, 48 (1982).

[10] D. Bevan, I. Grey, and B. Willis, J. Solid State Chem. 61, 1 (1986).

[11] D. A. Andersson, J. Lezama, B. P. Uberuaga, C. Deo, and S. D. Conradson, Phys. Rev. B 79, 024110 (2009).

[12] D. A. Andersson, G. Baldinozzi, L. Desgranges, D. R. Conradson, and S. D. Conradson, Inorg. Chem. 52, 2769 (2013).

[13] L. Desgranges, M. Gramond, C. Petot, G. Petot-Ervas, P. Ruello, and B. Saadi, J. Eur. Ceram. Soc. 25, 2683 (2005).

[14] A. Lidiard, J. Nucl. Mater. 19, 106 (1966).

[15] H. Matzke, J. Chem. Soc., Faraday Trans. 2 83, 1121 (1987).

[16] J. P. Crocombette, F. Jollet, L. T. Nga, and T. Petit, Phys. Rev. B 64, 104107 (2001). 
[17] J.-P. Crocombette, D. Torumba, and A. Chartier, Phys. Rev. B 83, 184107 (2011).

[18] P. Garcia, E. Pizzi, B. Dorado, D. Andersson, J.-P. Crocombette, C. Martial, G. Baldinozzi, D. Siméone, S. Maillard, and G. Martin, J. Nucl. Mater. 494, 461 (2017).

[19] F. Bruneval and J.-P. Crocombette, Phys. Rev. B 86, 140103 (2012).

[20] F. Bruneval, C. Varvenne, J.-P. Crocombette, and E. Clouet, Phys. Rev. B 91, 024107 (2015).

[21] R. M. Martin, Electronic Structure: Basic Theory and Practical Methods, Vol. 1 (Cambridge University Press, Cambridge, U.K., 2004).

[22] F. Bruneval, J.-P. Crocombette, X. Gonze, B. Dorado, M. Torrent, and F. Jollet, Phys. Rev. B 89, 045116 (2014).

[23] A. I. Liechtenstein, V. I. Anisimov, and J. Zaanen, Phys. Rev. B 52, R5467 (1995).

[24] S. L. Dudarev, G. A. Botton, S. Y. Savrasov, C. J. Humphreys, and A. P. Sutton, Phys. Rev. B 57, 1505 (1998).

[25] S. W. de Leeuw, J. W. Perram, and E. R. Smith, Proc. R. Soc. London Ser. A 373, 27 (1980).

[26] C. Freysoldt, J. Neugebauer, and C. G. Van de Walle, Phys. Rev. Lett. 102, 016402 (2009).

[27] S. E. Taylor and F. Bruneval, Phys. Rev. B 84, 075155 (2011).

[28] C. G. Van de Walle and R. M. Martin, Phys. Rev. Lett. 62, 2028 (1989).

[29] Y.-H. Li, X. G. Gong, and S.-H. Wei, Appl. Phys. Lett. 88, 042104 (2006).

[30] J. Wiktor, F. Bruneval, and A. Pasquarello, J. Chem. Theory Comput. 13, 3427 (2017).

[31] J. F. Janak, Phys. Rev. B 18, 7165 (1978).

[32] T. Petit, C. Lemaignan, F. Jollet, B. Bigot, and A. Pasturel, Philos. Mag. B 77, 779 (1998).

[33] F. Gupta, G. Brillant, and A. Pasturel, Philos. Mag. 87, 2561 (2007).

[34] H. Y. Geng, Y. Chen, Y. Kaneta, M. Iwasawa, T. Ohnuma, and M. Kinoshita, Phys. Rev. B 77, 104120 (2008).

[35] H. Y. Geng, Y. Chen, Y. Kaneta, and M. Kinoshita, Appl. Phys. Lett. 93, 201903 (2008).

[36] P. Nerikar, T. Watanabe, J. S. Tulenko, S. R. Phillpot, and S. B. Sinnott, J. Nucl. Mater. 384, 61 (2009).
[37] J. Yu, R. Devanathan, and W. J. Weber, J. Phys.: Condens. Matter 21, 435401 (2009).

[38] B. Dorado, G. Jomard, M. Freyss, and M. Bertolus, Phys. Rev. B 82, 035114 (2010).

[39] J.-P. Crocombette, Phys. Rev. B 85, 144101 (2012).

[40] B. Dorado, M. Freyss, B. Amadon, M. Bertolus, G. Jomard, and P. Garcia, J. Phys. Condens. Matter 25, 333201 (2013).

[41] E. Vathonne, J. Wiktor, M. Freyss, G. Jomard, and M. Bertolus, J. Phys.: Condens. Matter 26, 325501 (2014).

[42] J. Wang, R. C. Ewing, and U. Becker, Sci. Rep. 4, 4216 (2014).

[43] P. A. Burr and M. W. D. Cooper, Phys. Rev. B 96, 094107 (2017).

[44] G. Kresse and J. Furthmüller, Phys. Rev. B 54, 11169 (1996).

[45] P. E. Blöchl, Phys. Rev. B 50, 17953 (1994).

[46] A. Kotani and T. Yamazaki, Prog. Theor. Phys. 108, 117 (1992).

[47] B. Dorado, B. Amadon, M. Freyss, and M. Bertolus, Phys. Rev. B 79, 235125 (2009).

[48] R. Laskowski, G. K. H. Madsen, P. Blaha, and K. Schwarz, Phys. Rev. B 69, 140408 (2004).

[49] D. Gryaznov, E. Heifets, and D. Sedmidubsky, Phys. Chem. Chem. Phys. 12, 12273 (2010).

[50] J. C. Boettger, Eur. Phys. J. B 36, 15 (2003).

[51] J. P. Perdew, K. Burke, and M. Ernzerhof, Phys. Rev. Lett. 77, 3865 (1996).

[52] H. Hoekstra, A. Santoro, and S. Siegel, J. Inorg. Nucl. Chem. 18, 166 (1961).

[53] B. Willis, J. Phys. France 25, 431 (1964).

[54] N. Ashcroft and N. Mermin, Solid State Physics (Holt, Rinehart and Winston, New York, 1976).

[55] D. Labroche, O. Dugne, and C. Chatillon, J. Nucl. Mater. 312, 21 (2003).

[56] E. Moore, C. Guéneau, and J.-P. Crocombette, J. Solid State Chem. 203, 145 (2013).

[57] B. Dorado, D. A. Andersson, C. R. Stanek, M. Bertolus, B. P. Uberuaga, G. Martin, M. Freyss, and P. Garcia, Phys. Rev. B 86, 035110 (2012).

[58] J. Marin and P. Contamin, J. Nucl. Mater. 30, 16 (1969).

[59] H. Matzke, J. Nucl. Mater. 30, 26 (1969).

[60] G. Manhes, C. J. Allègre, B. Dupré, and B. Hamelin, Earth Planet. Sci. Lett. 47, 370 (1980). 